\title{
Structural-Electrical-Coupled Formulation for the Free Vibration of a Piezoelectric-Laminated Plate Using the Analytical Arbitrary Quadrilateral $p$ Element
}

\author{
Y. Y. Lee, A. Y. T. Leung, and B. Zhu \\ Department of Civil and Architectural Engineering, City University of Hong Kong, Hong Kong \\ Correspondence should be addressed to Y. Y. Lee, bcraylee@cityu.edu.hk
}

Received 7 November 2011; Revised 13 February 2012; Accepted 14 February 2012

Academic Editor: Svatoslav Staněk

Copyright (C) 2012 Y. Y. Lee et al. This is an open access article distributed under the Creative Commons Attribution License, which permits unrestricted use, distribution, and reproduction in any medium, provided the original work is properly cited.

\begin{abstract}
An analytical quadrilateral $p$ element is developed for solving the free vibrations of piezoelectriclaminated plates. The formulations of the displacement and strain fields are based on first-order shear deformation plate theory. The coupling effect between the electrical and stress fields is also considered. The Legendre orthogonal polynomials are used as the element interpolation functions, and the analytical integration technique is adopted. It is found that the present $p$ element method gives high numerical precision results, fast and monotonic convergence rate. In the numerical cases, the effects of the number of hierarchical terms and mesh size on the convergence rate are investigated. Examples of square plates with different displacement and potential boundary conditions are studied. In the comparisons, the solutions of the present element are in good agreement with those obtained from other classical and finite element methods.
\end{abstract}

\section{Introduction}

Various beam/plate problems have been hot research topics for decades (e.g., [1-15]), and many of them are solved using the finite element method (e.g., [16-21]). Beams/plates embedded with piezoelectric materials are widely used in various intelligent material systems for active control because of the electromechanical-coupling properties. For cases of simple geometric shapes, the two-dimensional (2D) and three-dimensional (3D) analytical methods that were developed by Benjeddou and Deü [22], Correia et al. [23], Heyliger and Saravanos [24], and Ding and Chen [25] are effective in analyzing the free vibrations of piezoelectric-laminated plates and provide clear physical meanings in the analytical and symbolical formulations. For cases of complex geometries, boundary conditions, and 
loadings, the finite element method (FEM) is more powerful and versatile than classical solutions. Most of the classical continuum solutions of plates have been limited to singleor two-mode approximations. This is due to the difficulties in obtaining the general multiple mode governing equations using Galerkin's approach, especially for plates with complex boundary conditions. Hence, Saravanos et al., Lam et al., He et al., and Loja et al. [2630] developed the finite-element and finite-strip methods for complicated piezoelectriclaminated plates. There are two main kinds of theories used for finite-element formulations. One is the classical plate theory (CPT), and the other one is the shear deformation theory, which branches out into first-order shear deformation theory (FSDT) and higher order shear deformation theory (HSDT). In general, theories that consider the shear deformation effect are more accurate for the analysis of piezoelectric-laminated plates. Thus, one such theory is used in this paper. The performance of an FEM model can be improved by a finer mesh ( $h$-version) or refined integrated piezoelectric sensor and actuator interpolation ( $p$-version). Zienkiewicz and Taylor [31] mentioned that the convergence rates of $p$-version elements would be more rapid than those of $h$-version elements for the same degrees of freedom (DOFs). The $p$-version elements that were developed by Houmat [32] and Woo et al. [33] were demonstrated to have fast monotonic convergence for cases of triangular plates and thick skew plates. According to the free-vibration analysis of skew Mindlin plates that Leung and Zhu [34] conducted, if the $p$-version approach is employed, then analytical integration is preferred in the procedures of setting up the element formulation; otherwise, the numerical integration errors may adversely affect the accuracy, and monotonic convergence of the natural frequencies cannot be guaranteed. In the axial free vibration analyses of beams, Leung and Chan [35] adopted trigonometric interpolation functions and the analytical integration technique to improve the accuracy. The analytical $p$ element approach has been further applied to various vibrating plate problems [36-39] such as membrane vibration and thick plates and laminated plates. Although this kind of element can be applied to the analysis of plates with triangular and other complicated shapes, it is tedious to set up the mesh and assemble the elements when compared with quadrilateral elements.

Based on FSDT, the quadrilateral hierarchical element is developed for the free vibrations of piezoelectric-laminated plates in this paper. Legendre orthogonal polynomials are adopted in the interpolation functions to improve the accuracy. Comparisons with published results and case studies show the good accuracy and efficiency of the present quadrilateral $p$ element.

\section{Theoretical Formulation}

\subsection{Analytical Model}

Figure 1 shows a laminated plate and the coordinate system defined at the midplane. The fiber direction is indicated by an angle $\theta$, which is the positive-rotation angle of the principal material axes from the arbitrary $x y$ axes. The elasticity modulus for a layer parallel to the fibers is $E_{11}$, and perpendicular to the fibers is $E_{22}$. The layers can be piezoelectric or of composite materials. It is assumed that the potential of the piezoelectric layer varies linearly through its thickness.

\subsection{Displacements and Strains}

According to FSDT [40], the displacement fields of the plate are expressed as: 


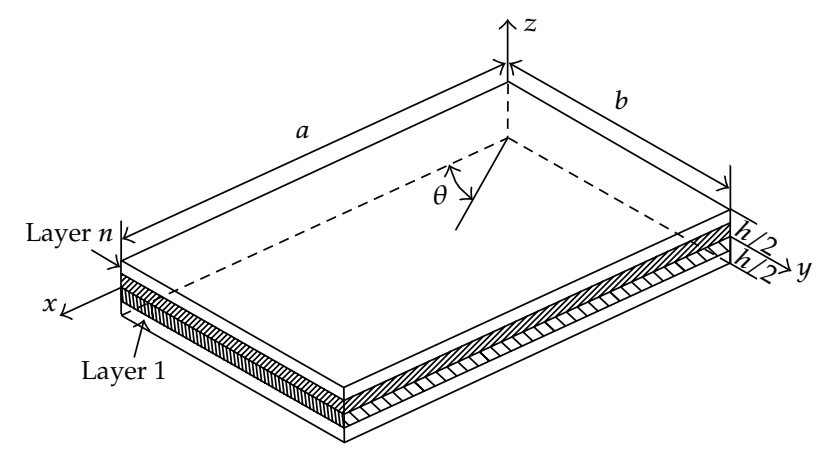

Figure 1: The configuration of a laminated composite plate.

$$
\begin{gathered}
\bar{u}(x, y, z)=u(x, y)+z \psi_{x}(x, y) \\
\bar{v}(x, y, z)=v(x, y)+z \psi_{y}(x, y) \\
\bar{w}(x, y, z)=w(x, y)
\end{gathered}
$$

where $\bar{u}, \bar{v}$, and $\bar{w}$ are the displacement components along the $x, y$, and $z$ axes, respectively, $u, v$, and $w$ are the associated midplane displacements, and $\psi_{x}, \psi_{y}$ are the normal rotations about the $y$ and $x$ axes, respectively.

The linear strain-displacement relations are given by

$$
\begin{gathered}
\varepsilon_{x}=\frac{\partial u}{\partial x}+z \frac{\partial \psi_{x}}{\partial x} \\
\varepsilon_{y}=\frac{\partial v}{\partial y}+z \frac{\partial \psi_{y}}{\partial y} \\
r_{x y}=\frac{\partial u}{\partial y}+\frac{\partial v}{\partial x}+z \frac{\partial \psi_{x}}{\partial y}+z \frac{\partial \psi_{y}}{\partial x} \\
r_{y z}=\frac{\partial w}{\partial y}+\psi_{y} \\
r_{x z}=\frac{\partial w}{\partial x}+\psi_{x} .
\end{gathered}
$$

The electrical potential is assumed to be linear through the thickness in each piezoelectric layer. Hence, the potential of the $k$ th layer $\phi$ can be expressed as

$$
\phi(x, y, z)=\frac{h^{k+1}-z}{h^{k+1}-h^{k}} \phi_{k}(x, y)+\frac{z-h^{k}}{h^{k+1}-h^{k}} \phi_{k+1}(x, y)
$$

where $\phi_{k}, \phi_{k+1}$ are the electrical potentials of the bottom and top surfaces of the $k$ th layer, respectively, and $h^{k}$ and $h^{k+1}$ are the $z$-coordinates corresponding to the bottom and top surfaces. 
Therefore, the electric field in the $k$ th layer can be written as:

$$
\mathbf{E}^{k}=-\nabla \phi=-\frac{1}{h^{k+1}-h^{k}}\left[\begin{array}{cc}
\left(h^{k+1}-z\right) \frac{\partial}{\partial x} & \left(z-h^{k}\right) \frac{\partial}{\partial x} \\
\left(h^{k+1}-z\right) \frac{\partial}{\partial y} & \left(z-h^{k}\right) \frac{\partial}{\partial y} \\
-1 & 1
\end{array}\right]\left\{\begin{array}{c}
\phi_{k} \\
\phi_{k+1}
\end{array}\right\}
$$

In two-dimensional problems, the transverse displacement $w$ can be interpolated using the following equation (2.5):

$$
w(\xi, \eta)=\sum_{k=1}^{p+2} \sum_{l=1}^{q+2} w_{k, l} N_{k, l}(\xi, \eta)
$$

where $N_{k, l}(\xi, \eta)=f_{k}(\xi) f_{l}(\eta) ; f_{k}(\xi)$ and $f_{l}(\eta)$ are $C^{0}$ Legendre orthogonal polynomials [40] (which are also used in the interpolations of displacements $u, v, \psi_{x}, \psi_{y}$ ).

When $k, l=1$ and 2, (2.5) represents a typical FEM interpolation. The hierarchical shape functions, when $k$ or $l>2$, lead to zero displacement at the corner nodes. Additional DOFs appear along the four edges and in the interior of the element. The DOFs at the four corner nodes are represented by $k$ and $l \leq 2$, and the DOFs along the four edges are represented by $k$ or $l>2$. Finally, the DOFs in the interior are represented by $k$ and $l>2$.

\subsection{Constitutive Relationships}

The constitutive relationships for the lamina-oriented arbitrarily, taking into account the piezoelectric effects are given as follows [25]:

$$
\begin{gathered}
\sigma=\overline{\mathbf{Q}} \varepsilon-\overline{\mathbf{e}}^{\mathrm{T}} \mathrm{E}, \\
\mathbf{D}=\overline{\mathbf{e}} \boldsymbol{\varepsilon}+\mathbf{p E}
\end{gathered}
$$

where $\boldsymbol{\sigma}$ is the stress matrix, $\boldsymbol{\varepsilon}$ is the strain vector, $\mathbf{D}$ is the electrical displacement vector, $\mathbf{E}$ is the electric field vector, $\overline{\mathbf{Q}}$ is the transformed elastic stiffness coefficient matrix, the coefficients of which are explicitly given by Reddy [41], $\mathbf{p}$ is the permittivity matrix, and $\overline{\mathbf{e}}$ is the transformed piezoelectric stress coefficient matrix, which is given by

$$
\begin{aligned}
& \bar{e}_{31}=e_{31} \cos ^{2} \theta+e_{32} \sin ^{2} \theta, \\
& \bar{e}_{32}=e_{31} \sin ^{2} \theta+e_{32} \cos ^{2} \theta, \\
& \bar{e}_{14}=\left(e_{15}-e_{24}\right) \sin \theta \cos \theta, \\
& \bar{e}_{15}=e_{15} \cos ^{2} \theta+e_{24} \sin ^{2} \theta, \\
& \bar{e}_{24}=e_{24} \cos ^{2} \theta+e_{15} \sin ^{2} \theta,
\end{aligned}
$$




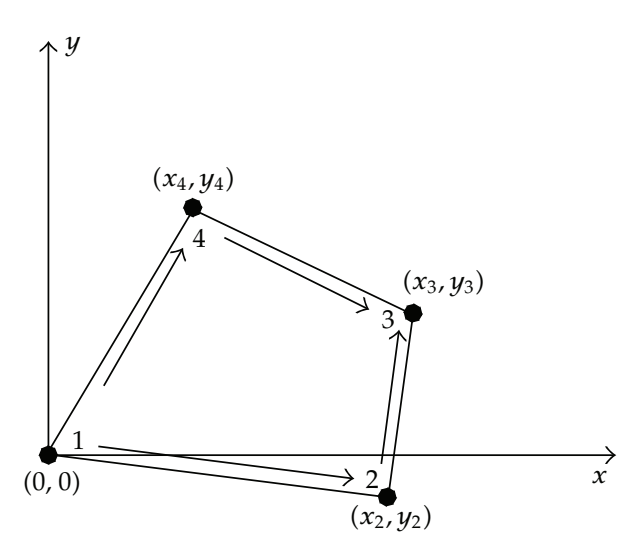

(a)

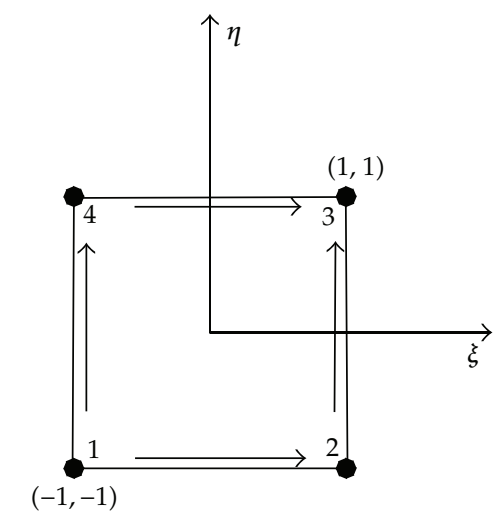

(b)

Figure 2: The coordinate transformation of a quadrilateral element.

$$
\begin{aligned}
& \bar{e}_{25}=\left(e_{15}-e_{24}\right) \sin \theta \cos \theta, \\
& \bar{e}_{36}=\left(e_{31}-e_{32}\right) \sin \theta \cos \theta,
\end{aligned}
$$

where $e_{i j}$ are the piezoelectric stress coefficients in the directions parallel and perpendicular to the fibers, and $\theta$ is the fiber direction.

\subsection{Finite Element Model}

The coordinate systems that are used to define an arbitrary quadrilateral plate element are shown in Figures 1 and 2 depict the Cartesian plane coordinate system of the element and the mapped $\xi-\eta$ square plane region. The Jacobian matrix is expressed in terms of the Cartesian coordinates at the four corner nodes:

$$
\mathbf{J}=\left[\begin{array}{ll}
\frac{\partial x}{\partial \xi} & \frac{\partial y}{\partial \xi} \\
\frac{\partial x}{\partial \eta} & \frac{\partial y}{\partial \eta}
\end{array}\right]=\left[\begin{array}{ll}
a+b \eta & d+e \eta \\
c+b \xi & f+e \xi
\end{array}\right]
$$

where $a=0.25\left(x_{2}+x_{3}-x_{4}\right), b=0.25\left(-x_{2}+x_{3}-x_{4}\right), c=0.25\left(-x_{2}+x_{3}+x_{4}\right), d=0.25\left(y_{2}+y_{3}-y_{4}\right)$, $e=0.25\left(-y_{2}+y_{3}-y_{4}\right)$, and $f=0.25\left(-y_{2}+y_{3}+y_{4}\right)$.

The determinant of the Jacobian matrix is $|\mathbf{J}|=(a e-b d) \xi+(b f-c e) \eta+a f-c d$, and

$$
\mathbf{J}^{-1}=\frac{1}{|\mathbf{J}|}\left[\begin{array}{cc}
f+e \xi & -(d+e \eta) \\
-(c+b \xi) & a+b \eta
\end{array}\right] .
$$


The displacement fields $u, v, w, \psi_{x}$, and $\psi_{y}$ and the electric potential $\phi^{k}$ are interpolated using the $C^{0}$ Legendre orthogonal polynomials as

$$
\begin{gathered}
\mathbf{u}=\left\{\begin{array}{c}
u \\
v \\
w \\
\psi_{x} \\
\psi_{y}
\end{array}\right\}=\left[N_{1,1} \mathbf{I}, N_{1,2} \mathbf{I}, \ldots, N_{1, q+2} \mathbf{I}, N_{2,1} \mathbf{I}, \ldots, N_{p+2, q+2} \mathbf{I}\right] \boldsymbol{\delta}^{e}=\mathbf{N}_{u} \cdot \boldsymbol{\delta}^{e}, \\
\phi_{k}=\left[N_{1,1}, N_{1,2}, \ldots, N_{1, q+2}, N_{2,1}, \ldots, N_{p+2, q+2}\right] \phi_{k}^{e}=\mathbf{N}_{\phi} \cdot \phi_{k^{\prime}}^{e}
\end{gathered}
$$

where $\delta^{e}$ is the vector of generalized DOFs, $\phi_{e}^{k}$ is the nodal electric potential in the bottom surface of the $k$ th layer, and $\mathbf{I}$ is the $5 \times 5$ identity matrix. Substituting the expressions of the displacement fields into (2.2) and (2.4) gives

$$
\begin{gathered}
\boldsymbol{\varepsilon}=\left\{\begin{array}{l}
\varepsilon_{x} \\
\varepsilon_{y} \\
r_{x y} \\
r_{y z} \\
r_{x z}
\end{array}\right\}=\left[\begin{array}{ccccc}
\frac{\partial}{\partial x} & 0 & 0 & \frac{z \partial}{\partial x} & 0 \\
0 & \frac{\partial}{\partial y} & 0 & 0 & \frac{z \partial}{\partial y} \\
\frac{\partial}{\partial y} & \frac{\partial}{\partial x} & 0 & \frac{z \partial}{\partial y} & \frac{z \partial}{\partial x} \\
0 & 0 & \frac{\partial}{\partial x} & 1 & 0 \\
0 & 0 & \frac{\partial}{\partial y} & 0 & 1
\end{array}\right] \mathbf{u}=\mathbf{B}_{u} \cdot \boldsymbol{\delta}^{e}, \\
\mathbf{E}^{k}=\left\{\begin{array}{l}
E_{x} \\
E_{y} \\
E_{z}
\end{array}\right\}=-\frac{1}{h^{k+1}-h^{k}}\left[\begin{array}{ccc}
\left(h^{k+1}-z\right) \frac{\partial}{\partial x} & \left(z-h^{k}\right) \frac{\partial}{\partial x} \\
\left(h^{k+1}-z\right) \frac{\partial}{\partial y} & \left(z-h^{k}\right) \frac{\partial}{\partial y} \\
-1 & 1
\end{array}\right]\left\{\begin{array}{c}
\phi_{k} \\
\phi_{k+1}
\end{array}\right\}=-\mathbf{B}_{\phi} \cdot \mathbf{\Phi}^{e},
\end{gathered}
$$

where $\boldsymbol{\Phi}^{e}=\left[\phi_{1}^{e}, \ldots, \phi_{k}^{e}, \phi_{k+1}^{e}, \ldots, \phi_{n+1}^{e}\right]^{\mathrm{T}}, n$ is the total number of layers.

The potential energies of the element include the elastic strain energy, the piezoelectric energy, and the electrical energy, which are given as

$$
\begin{aligned}
H & =\frac{1}{2} \sum_{k=1}^{n} \int_{h_{k}}^{h_{k+1}}\left[\iint\left(\varepsilon^{T} \overline{\mathbf{Q}} \boldsymbol{\varepsilon}-2 \varepsilon^{T} \overline{\mathbf{e}}^{T} \mathbf{E}-\mathbf{E}^{T} \mathbf{p} \mathbf{E}\right) d x d y\right] d z \\
& =\frac{1}{2} \boldsymbol{\delta}^{e T} \sum_{k=1}^{n} \int_{h_{k}}^{h_{k+1}}\left[\iint \mathbf{B}_{u}^{T} \overline{\mathbf{Q}} \mathbf{B}_{u} d x d y\right] d z \cdot \boldsymbol{\delta}^{e}
\end{aligned}
$$


Abstract and Applied Analysis

$$
\begin{aligned}
& +\boldsymbol{\delta}^{e T} \sum_{k=1}^{n} \int_{h_{k}}^{h_{k+1}}\left[\iint \mathbf{B}_{u}^{T} \overline{\mathbf{e}}^{T} \mathbf{B}_{\phi} d x d y\right] d z \cdot \mathbf{\Phi}^{e} \\
& -\frac{1}{2} \mathbf{\Phi}^{e T} \sum_{k=1}^{n} \int_{h_{k}}^{h_{k+1}}\left[\iint \mathbf{B}_{\phi}^{T} \mathbf{p} \mathbf{B}_{\phi} d x d y\right] d z \cdot \mathbf{\Phi}^{e} .
\end{aligned}
$$

The kinetic energy of the element is given by

$$
T^{e}=\frac{1}{2} \sum_{k=1}^{n} \int_{h_{k}}^{h_{k+1}}\left\{\iint\left[\rho\left(\dot{u}^{2}+\dot{v}^{2}+\dot{w}^{2}\right)+z^{2} \rho\left(\dot{\psi}_{x}^{2}+\dot{\psi}_{y}^{2}\right)\right] d x d y\right\} d z
$$

The Hamilton principle is adopted in the derivation of the equation of the eigenvalue problem, which is as follows:

$$
\left(\left[\begin{array}{ll}
\mathbf{K}_{u u} & \mathbf{K}_{u \phi} \\
\mathbf{K}_{\phi u} & \mathbf{K}_{\phi \phi}
\end{array}\right]-\omega^{2}\left[\begin{array}{cc}
\mathbf{M}_{u u} & 0 \\
\mathbf{0} & 0
\end{array}\right]\right)\left\{\begin{array}{l}
\boldsymbol{\delta} \\
\phi
\end{array}\right\}=\mathbf{0}
$$

where

$$
\begin{aligned}
\mathbf{K}_{u u} & =\sum_{e} \mathbf{K}_{u u}^{e}, \quad \mathbf{K}_{u \phi}=\sum_{e} \mathbf{K}_{u \phi}^{e}, \quad \mathbf{M}_{u u}=\sum_{e} \mathbf{M}_{u u^{\prime}}^{e} \quad \mathbf{K}_{\phi u}=\mathbf{K}_{u \phi^{\prime}}^{T} \\
\mathbf{K}_{u u}^{e} & =\sum_{k=1}^{n} \int_{h_{k}}^{h_{k+1}} \iint \mathbf{B}_{u}^{T} \overline{\mathbf{Q}} \mathbf{B}_{u} d x d y d z=\sum_{k=1}^{n} \int_{h_{k}}^{h_{k+1}} \int_{-1}^{1} \int_{-1}^{1} \mathbf{B}_{u}^{T} \overline{\mathbf{Q}} \mathbf{B}_{u} \cdot|\mathbf{J}| d \xi d \eta d z, \\
\mathbf{K}_{u \phi}^{e} & =\sum_{k=1}^{n} \int_{h_{k}}^{h_{k+1}} \iint \mathbf{B}_{u}^{T} \overline{\mathbf{e}}^{T} \mathbf{B}_{\phi} d x d y d z=\sum_{k=1}^{n} \int_{h_{k}}^{h_{k+1}} \int_{-1}^{1} \int_{-1}^{1} \mathbf{B}_{u}^{T} \overline{\mathbf{e}}^{T} \mathbf{B}_{\phi} \cdot|\mathbf{J}| \mathrm{d} \xi d \eta d z, \\
\mathbf{K}_{\phi \phi}^{e} & =\sum_{k=1}^{n} \int_{h_{k}}^{h_{k+1}} \iint \mathbf{B}_{\phi}^{T} \mathbf{p} \mathbf{B}_{\phi} d x d y d z=\sum_{k=1}^{n} \int_{h_{k}}^{h_{k+1}} \int_{-1}^{1} \int_{-1}^{1} \mathbf{B}_{\phi}^{T} \mathbf{p} \mathbf{B}_{\phi} \cdot|\mathbf{J}| d \xi d \eta d z, \\
\mathbf{M}_{u u}^{e}= & \sum_{k=1}^{n} \int_{h_{k}}^{h_{k+1}} \iint \rho \cdot \mathbf{N}^{T} \operatorname{diag}\left[1,1,1, z^{2}, z^{2}\right] \mathbf{N} d x d y d z \\
& =\sum_{k=1}^{n} \int_{h_{k}}^{h_{k+1}} \int_{-1}^{1} \int_{-1}^{1} \rho \cdot \mathbf{N}^{T} \operatorname{diag}\left[1,1,1, z^{2}, z^{2}\right] \mathbf{N} \cdot|\mathbf{J}| d \xi d \eta d z .
\end{aligned}
$$

In the procedure of coordinate mapping, the derivative in $\mathbf{B}_{u}$ and $\mathbf{B}_{\phi}$ should be replaced by

$$
\left\{\begin{array}{l}
\frac{\partial}{\partial x} \\
\frac{\partial}{\partial y}
\end{array}\right\}=\mathbf{J}^{-1}\left\{\begin{array}{c}
\frac{\partial}{\partial \xi} \\
\frac{\partial}{\partial \eta}
\end{array}\right\} .
$$




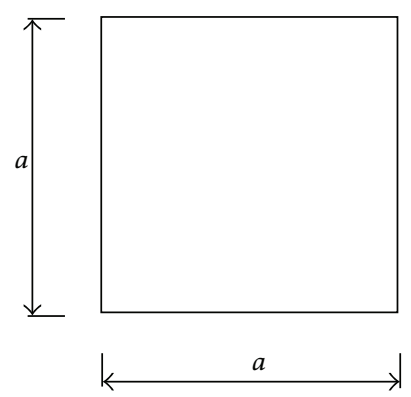

(a)
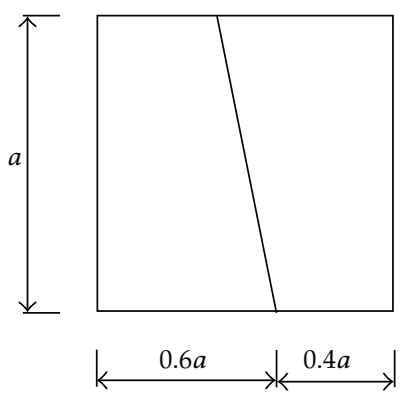

(b)

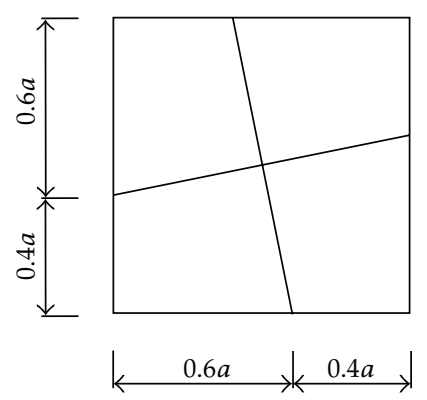

(c)

Figure 3: The 3 meshes adopted for the square plate.

Table 1: Elastic, piezoelectric, and dielectric properties of the materials.

\begin{tabular}{cccccccc}
\hline Property & G-1195N & Ti-6A1-4V & PZT-4 & Aluminum & G/E & Property & PZT-5H \\
\hline$E_{1}(\mathrm{Gpa})$ & 63 & 105.7 & 81.3 & 68.3 & 132.38 & $c_{11}^{E}(\mathrm{GPa})$ & 126 \\
$E_{2}$ & 63 & 105.7 & 81.3 & 68.3 & 10.756 & $c_{33}^{E}$ & 117 \\
$v_{12}$ & 0.3 & 0.2981 & 0.329 & 0.3 & 0.24 & $c_{44}^{E}$ & 230 \\
$G_{23}$ & 24.23 & 40.71 & 25.6 & & 3.606 & $c_{66}^{E}$ & 23.5 \\
$G_{31}$ & 24.23 & 40.71 & 25.6 & & 5.6537 & $c_{12}^{E}$ & 79.5 \\
$G_{12}$ & 24.23 & 40.71 & 30.6 & & 5.6537 & $c_{13}^{E}$ & 84.1 \\
$e_{24}\left(\mathrm{C} / \mathrm{m}^{2}\right)$ & & & 12.72 & & & $\varepsilon_{33}^{S} / \varepsilon_{0}$ & 1700 \\
$e_{31}$ & 22.86 & & -5.20 & & & $\left(\mathrm{C} / \mathrm{m}^{2}\right)$ & \\
$e_{32}$ & 22.86 & & -5.20 & & & $\varepsilon_{11}^{s} / / \varepsilon_{0}$ & 1400 \\
$p_{11} / p_{0}$ & 1695 & & 1475 & & 3.5 & $e_{31}\left(\mathrm{~F} / \mathrm{m}^{2}\right)$ & -6.55 \\
$p_{22} / p_{0}$ & 1695 & & 1475 & & 3.0 & $e_{33}$ & 23.3 \\
$p_{33} / p_{0}$ & 1695 & & 1300 & & 3.0 & & 17 \\
$\rho\left(\mathrm{kg} / \mathrm{m}^{3}\right)$ & 7600 & 4429 & 7600 & 2698 & 1578 & $\rho\left(\mathrm{kg} / \mathrm{m}^{3}\right)$ & 7500 \\
\hline
\end{tabular}

Note that analytic integration is adopted in (2.16). Hence, the analytic integrations of the stiffness and mass matrices are in the form of $\xi^{i} \eta^{j} /(A \xi+B \eta+C)$, where $A, B$, and $C$ are constants.

\section{Numerical Results and Discussions}

In this section, case studies are carried out to show the performance of the quadrilateral $p$ element. The elastic, piezoelectric, and dielectric material properties that are adopted in the case studies are given in Table 1 . There are two sets of electric boundary conditions for the outer surface of the piezoelectric layers: a closed circuit condition $\Phi=0$, (i.e., the potential is grounded) and an open circuit condition $D_{z}=0$, (i.e., the electric displacement is zero). Unless otherwise stated, the number of the hierarchical term $p$ is 4, and the mesh in Figure 3(c) is used. 

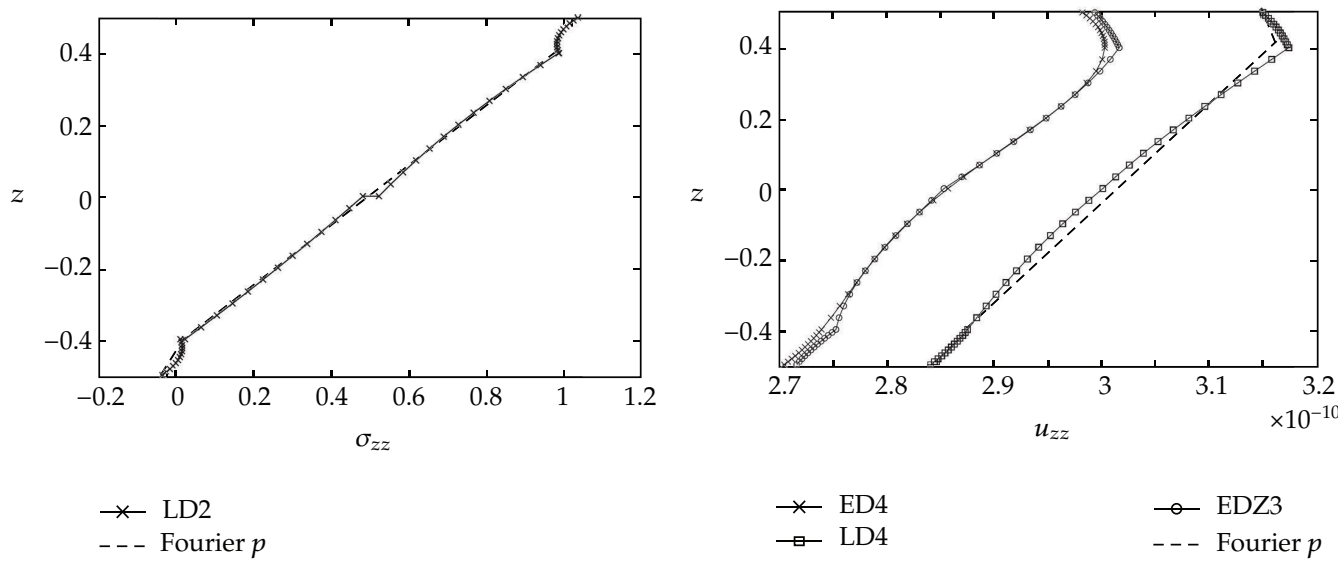

$\begin{array}{ll}* & \text { LD2 } \\ --- & \text { Fourier } p\end{array}$

$*$ ED
$\rightarrow \mathrm{LD} 4$

-- - Fourier $p$

(a)

(b)

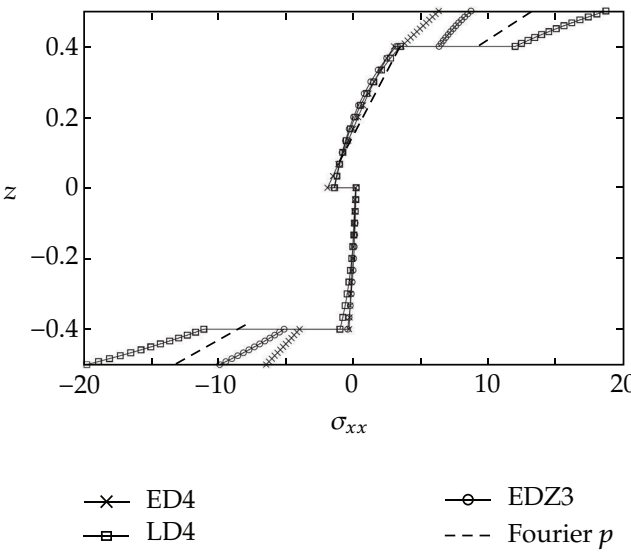

(c)

Figure 4: The comparisons between (a) the transverse normal stress, (b) transverse displacement, and (c) in-plane stress results of a sensor plate from the proposed method and [8].

Table 2: Fundamental frequency parameter $\lambda\left(=\omega a^{2} \rho^{1 / 2} / 2000 \pi h\right)$ of a simply supported sandwich square plate $\left(\Phi=0, a / h=10, \rho=\rho_{i} h_{i} / h\right)$.

\begin{tabular}{lccc}
\hline Mesh & 1 rectangular & 2 quadrilateral & 4 quadrilateral \\
\hline$p=1$ & 230.930 & 228.647 & 224.015 \\
$p=2$ & 224.341 & 220.846 & 217.495 \\
$p=3$ & 217.327 & 217.333 & 217.294 \\
$p=4$ & 217.301 & 217.298 & 217.291 \\
$p=5$ & 217.291 & 217.291 & 217.291 \\
\hline 2D solution [22] & & 216.602 & \\
3D solution [42] & & 214.933 & \\
\hline
\end{tabular}

\subsection{Convergence Study of a Square Plate}

The free vibration of a simply supported hybrid sandwich square plate is investigated here. The accuracy of the present element can be seen in this convergence study. The lamina 
Table 3: Natural frequencies $(\mathrm{Hz})$ of a three-ply square plate $(a=0.4 m, \Phi=0)$.

\begin{tabular}{lcccccc}
\hline \multirow{2}{*}{\begin{tabular}{c} 
Mode No. \\
\cline { 3 - 6 }
\end{tabular}} & $\begin{array}{c}\text { Reference } \\
{[28]}\end{array}$ & This paper & $\begin{array}{c}\text { Reference } \\
{[28]}\end{array}$ & This paper & $\begin{array}{c}\text { Reference } \\
{[28]}\end{array}$ & This paper \\
\hline 1 & 144.25 & 142.05 & 262.53 & 258.16 & 25.78 & 24.56 \\
2 & 359.00 & 354.91 & 533.83 & 526.10 & 62.75 & 60.18 \\
3 & 359.00 & 354.92 & 533.83 & 526.14 & 157.20 & 150.54 \\
4 & 564.10 & 567.39 & 774.20 & 774.55 & 200.19 & 192.23 \\
5 & 717.80 & 710.20 & 957.32 & 948.70 & 228.22 & 218.80 \\
6 & 717.80 & 710.67 & 963.04 & 952.89 & 397.58 & 382.63 \\
7 & 908.25 & 922.13 & 1172.70 & 1183.11 & 452.26 & 433.06 \\
8 & 908.25 & 923.67 & 1172.70 & 1188.36 & 472.76 & 454.12 \\
9 & 1223.14 & 1208.09 & 1535.81 & 1522.02 & 522.91 & 501.67 \\
10 & 1223.14 & 1216.77 & 1535.81 & 1530.89 & 677.28 & 657.42 \\
\hline
\end{tabular}

Table 4: Nondimensional frequencies $\left(\omega a^{2} \rho^{1 / 2} / 2000 \pi h\right)$ of a simple supported square plate with different potential boundary conditions.

\begin{tabular}{lcccc}
\hline \multirow{2}{*}{ Mode No. } & \multicolumn{2}{c}{$\Phi=0$} \\
& Reference [22] & This paper & Reference [22] & This paper \\
\hline 1 & 246.07 & 236.96 & 246.07 & 234.97 \\
2 & 559.62 & 536.14 & 559.62 & 530.74 \\
3 & 693.61 & 678.30 & 693.60 & 674.10 \\
4 & 967.16 & 938.32 & 967.14 & 930.60 \\
5 & 1091.5 & 1051.69 & 1091.46 & 1040.93 \\
\hline
\end{tabular}

configuration is PZT-4/GE/PZT-4. The thicknesses of the layers are $0.1 \mathrm{~h}, 0.8 \mathrm{~h}, 0.1 \mathrm{~h}$, respectively. The close circuit condition is considered. The plate is meshed into one, two, or four quadrilateral elements, as shown in Figure 3. The fundamental frequency parameter is shown in Table 2 for different numbers of hierarchical terms. It can be observed that the monotonic convergence rate is very fast with respect to the number of hierarchical terms, and the results are in good agreement with those obtained from the 2D analytical method [22] and the 3D state space method [42]. It should be noted that the electrical potentials in [22, 42] are assumed to be quadratic through the plate thickness, whereas they are linear in the present method. Thus, there is a small difference between the results.

\subsection{Square Plates with Different Displacement Boundary Conditions}

The first ten frequencies of a three-ply piezoelectric-laminated square plate (G-1195N/Ti6A1-4V/G-1195N, $0.1 \mathrm{~mm} / 0.48 \mathrm{~mm} / 0.1 \mathrm{~mm}$ ) are computed for three boundary conditions: simply supported (SSSS), fully clamped (CCCC), and cantilevered (CFFF). The electric boundary condition is a closed circuit. He et al. [28] derived finite element solutions using a traditional laminated plate element and CPT. A comparison of the results of our element and those of the traditional elements is shown in Table 3. Besides, a comparison for a static case is shown in Figure 4. The transverse normal stress, transverse displacement, and in-plane stress results of the sensor plate from the proposed method reasonably agree with those from [8]. 
Table 5: Natural frequencies $(\mathrm{Hz})$ of the aluminum trapezoidal plates with a top and a bottom PZT-5H layers.

\begin{tabular}{|c|c|c|c|c|c|c|c|}
\hline$\overline{L_{c}} / L_{a}$ & $L_{b} / L_{a}$ & $\beta$ degree & Mode 1 & Mode 2 & Mode 3 & Mode 4 & Mode 5 \\
\hline & & & & & CFFF & & \\
\hline & & 0 & 973.25 & 3453.32 & 5008.23 & 8766.12 & 12533.2 \\
\hline \multirow[t]{3}{*}{0.4} & 1 & $16.70^{*}$ & 1012.18 & 3593.01 & 5054.03 & 9450.83 & 12505.7 \\
\hline & & 30 & 977.83 & 3471.64 & 5015.1 & 8848.56 & 12546.9 \\
\hline & & 0 & 254.19 & 1305.3 & 1603 & 3402.94 & 4021.24 \\
\hline \multirow[t]{3}{*}{0.4} & 2 & $8.53^{*}$ & 258.77 & 1335.07 & 1586.97 & 3499.12 & 3991.47 \\
\hline & & 30 & 2285.42 & 1149.58 & 1605.29 & 2970.13 & 4028.11 \\
\hline & & 0 & 897.68 & 2764.03 & 4863.96 & 7820.35 & 10630.2 \\
\hline \multirow[t]{3}{*}{0.6} & 1 & $11.31^{*}$ & 916 & 2805.25 & 4907.47 & 8118.05 & 10327.9 \\
\hline & & 30 & 856.46 & 2670.14 & 4742.59 & 7284.49 & 11111.1 \\
\hline & & 0 & 229 & 1218.28 & 1300.72 & 3295.31 & 3528.89 \\
\hline \multirow[t]{4}{*}{0.6} & 2 & $5.71^{*}$ & 231.29 & 1238.89 & 1289.27 & 3389.2 & 3460.19 \\
\hline & & 30 & 199.23 & 1028.21 & 1314.46 & 2697.62 & 3588.43 \\
\hline & & & & & FFFF & \multicolumn{2}{|c|}{ (exclude rigid body) } \\
\hline & & 0 & 3751.02 & 5081.51 & 7227.24 & 8855.43 & 12778.2 \\
\hline \multirow[t]{3}{*}{0.4} & 1 & $16.70^{*}$ & 4451.76 & 4829.61 & 6826.49 & 9817.23 & 12464.5 \\
\hline & & 30 & 3817.43 & 5072.35 & 7192.89 & 8949.32 & 12759.9 \\
\hline & & 0 & 1232.02 & 2244.2 & 3311.34 & 4476.95 & 6100.56 \\
\hline \multirow[t]{3}{*}{0.4} & 2 & $8.53^{*}$ & 1264.08 & 2241.91 & 3412.1 & 4483.82 & 6086.82 \\
\hline & & 30 & 1046.53 & 2225.88 & 2814.41 & 4341.84 & 5257.84 \\
\hline & & 0 & 3531.18 & 4795.26 & 6657.03 & 8273.77 & 11532.4 \\
\hline \multirow[t]{3}{*}{0.6} & 1 & $11.31^{*}$ & 3757.89 & 4763.2 & 6469.25 & 8660.78 & 11502.7 \\
\hline & & 30 & 3137.3 & 4788.39 & 6705.12 & 7939.43 & 11502.7 \\
\hline & & 0 & 1229.73 & 1884.67 & 3329.66 & 3938.8 & 5896.75 \\
\hline \multirow[t]{4}{*}{0.6} & 2 & $5.71^{*}$ & 1245.76 & 1880.09 & 3396.07 & 3915.9 & 5860.11 \\
\hline & & 30 & 973.25 & 1921.31 & 2571.67 & 3959.41 & 4738.01 \\
\hline & & & & & CCCC & & \\
\hline & & 0 & 11972.1 & 18409.3 & 23380.9 & 26724.3 & 31304.3 \\
\hline \multirow[t]{3}{*}{0.4} & 1 & $16.70^{*}$ & 11527.9 & 18338.3 & 22439.7 & 27022 & 30731.8 \\
\hline & & 30 & 11908 & 18391 & 23266.4 & 26747.2 & 31258.5 \\
\hline & & 0 & 8440.94 & 11415.7 & 14477.4 & 17653.6 & 18326.9 \\
\hline \multirow[t]{3}{*}{0.4} & 2 & $8.53^{*}$ & 8326.44 & 11312.6 & 14397.2 & 17678.8 & 17994.8 \\
\hline & & 30 & 9247.02 & 12159.9 & 15084.2 & 17999.4 & 20069.6 \\
\hline & & 0 & 9867.61 & 16105.6 & 20005.4 & 24434.3 & 26701.4 \\
\hline \multirow[t]{3}{*}{0.6} & 1 & $11.31^{*}$ & 9695.86 & 16169.7 & 19492.5 & 25235.8 & 25762.5 \\
\hline & & 30 & 10309.6 & 16075.8 & 21104.6 & 23884.7 & 27731.9 \\
\hline & & 0 & 7346.32 & 9395.87 & 11756.9 & 14912.5 & 16565.9 \\
\hline \multirow[t]{2}{*}{0.6} & 2 & $5.71^{*}$ & 7295.94 & 9350.07 & 11731.7 & 14930.8 & 16444.5 \\
\hline & & 30 & 8418.04 & 10405.8 & 12455.3 & 14958.3 & 17976.5 \\
\hline
\end{tabular}

${ }^{*}$ Symmetric trapezoidal plates.

\subsection{Square and Trapezoidal Plates with Different Potential Boundary Conditions}

To study the validity of the present element for different potential boundary conditions, a five-ply simply supported plate, which is made of three plies of G/E with the laminate sequence $\left(0^{\circ}, 90^{\circ}, 0^{\circ}\right)$ and two surface-bonded PZT-4 piezoelectric layers, is considered. The 


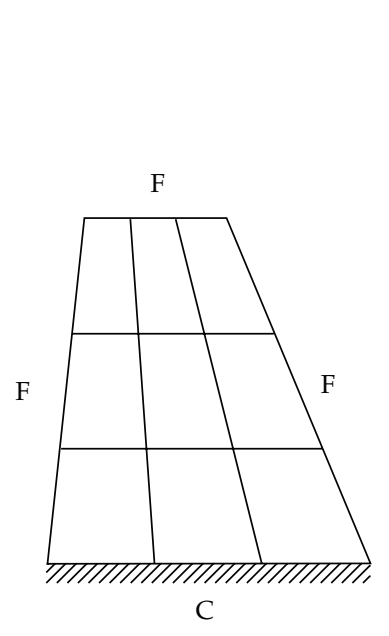

(a)
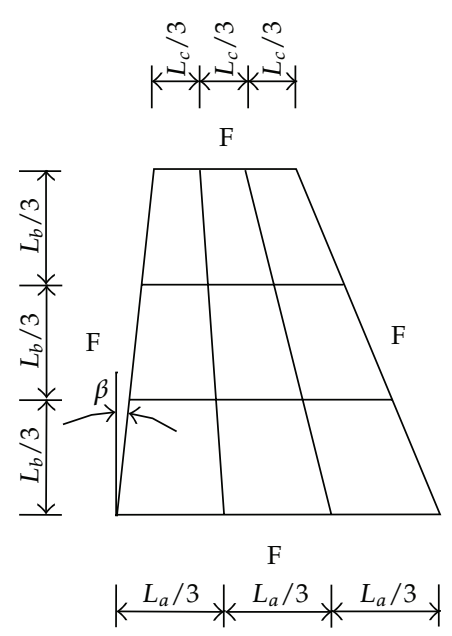

(b)

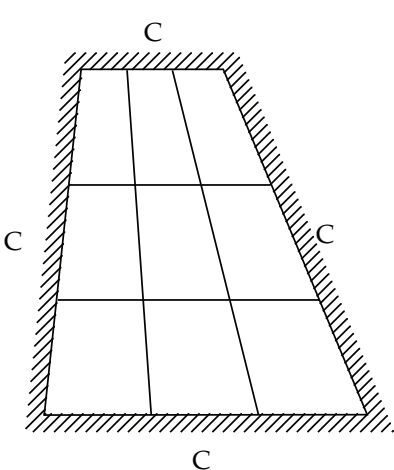

(c)

Figure 5: The 3 meshes adopted for the $10 \mathrm{~mm}$ aluminum trapezoidal plates with $1 \mathrm{~mm}$ PZT-5H layers perfectly bonded on both the upper and lower surfaces having various boundary conditions (the number of Fourier terms: $p=q=5, L_{a}=0.1 \mathrm{~m}$ ).

thickness of both piezoelectric layers is $0.1 \mathrm{~h}$, and all G/E layers have the same thickness. The unit density is adopted for fair comparison. The solutions of the present method are presented in Table 4 and are compared with the 3D exact solution. Besides, Figure 5 shows the trapezoidal plates with PZT-5H layers perfectly bonded on both the upper and lower surfaces having various boundary conditions (the number of Fourier terms used are $p=q=5$ ). The thicknesses of the trapezoidal plates and PZT-5H layers are $10 \mathrm{~mm}$ and $1 \mathrm{~mm}$, respectively. Table 5 shows the natural frequencies of the aluminum trapezoidal plates with PZT-5H layers.

\section{Conclusion}

A quadrilateral $p$ element with analytical integration for the free vibration of piezoelectriclaminated composite plates is presented. The Legendre orthogonal polynomials are used as the element interpolation functions, and the analytical integration technique is adopted. The monotonic convergence rate of the present element is very fast with respect to the number of hierarchical terms. Comparisons between the solutions that are obtained from our method and those of other methods show that the element is accurate and efficient for free-vibration analyses of piezoelectric-laminated plates.

\section{Acknowledgment}

The research described herein was supported by Grant number CityU SRG7008100.

\section{References}

[1] A. Borisovich and J. Janczewska, "Stable and unstable bifurcation in the von Kármán problem for a circular plate," Abstract and Applied Analysis, vol. 2005, no. 8, pp. 889-899, 2005. 
[2] M. L. Santos, J. Ferreira, and C. A. Raposo, "Existence and uniform decay for a nonlinear beam equation with nonlinearity of Kirchhoff type in domains with moving boundary," Abstract and Applied Analysis, vol. 2005, no. 8, pp. 901-919, 2005.

[3] J. A. Esquivel-Avila, "Dynamic analysis of a nonlinear Timoshenko equation," Abstract and Applied Analysis, Article ID 724815, 36 pages, 2011.

[4] Y. X. Li and H. Yang, "An existence and uniqueness result for a bending beam equation without growth restriction," Abstract and Applied Analysis, vol. 2010, Article ID 694590, 9 pages, 2010.

[5] M. Grasselli, V. Pata, and G. Prouse, "Longtime behavior of a viscoelastic Timoshenko beam," Discrete and Continuous Dynamical Systems A, vol. 10, no. 1-2, pp. 337-348, 2004.

[6] J. E. Muñoz Rivera and R. Racke, "Global stability for damped Timoshenko systems," Discrete and Continuous Dynamical Systems A, vol. 9, no. 6, pp. 1625-1639, 2003.

[7] E. Carrera, "An improved reissner-mindlin-type model for the electromechanical analysis of multilayered plates including piezo-layers," Journal of Intelligent Material Systems and Structures, vol. 8, no. 3, pp. 232-248, 1997.

[8] E. Carrera and M. Boscolo, "Classical and mixed finite elements for static and dynamic analysis of piezoelectric plates," International Journal for Numerical Methods in Engineering, vol. 70, no. 10, pp. 1135-1181, 2007.

[9] E. Carrera, S. Brischetto, and P. Nali, "Variational statements and computational models for multifield problems and multilayered structures," Mechanics of Advanced Materials and Structures, vol. 15, no. 3-4, pp. 182-198, 2008.

[10] E. Carrera and P. Nali, "Mixed piezoelectric plate elements with direct evaluation of transverse electric displacement," International Journal for Numerical Methods in Engineering, vol. 80, no. 4, pp. 403-424, 2009.

[11] E. Carrera and P. Nali, "Multilayered plate elements for the analysis of multifield problems," Finite Elements in Analysis and Design, vol. 46, no. 9, pp. 732-742, 2010.

[12] E. Carrera, S. Brischetto, and P. Nali, Plates and Shells for Smart Structures, John Wiley and Sons, New York, NY, USA, 2011.

[13] E. Carrera, S. Brischetto, and M. Cinefra, "Variable kinematics and advanced variational statements for free vibrations analysis of piezoelectric plates and shells," Computer Modeling in Engineering and Sciences, vol. 65, no. 3, pp. 259-341, 2010.

[14] S. Brischetto and E. Carrera, "Coupled thermo-mechanical analysis of one-layered and multilayered plates," Composite Structures, vol. 92, no. 8, pp. 1793-1812, 2010.

[15] E. Carrera, A. Büttner, and P. Nali, "Mixed elements for the analysis of anisotropic multilayered piezoelectric plates," Journal of Intelligent Material Systems and Structures, vol. 21, no. 7, pp. 701-717, 2010.

[16] A. Caboussat and R. Glowinski, "Numerical solution of a variational problem arising in stress analysis: the vector case," Discrete and Continuous Dynamical Systems A, vol. 27, no. 4, pp. 1447-1472, 2010.

[17] C. Thai-Hoang, N. Nguyen-Thanh, H. Nguyen-Xuan, and T. Rabczuk, "An alternative alpha finite element method with discrete shear gap technique for analysis of laminated composite plates," Applied Mathematics and Computation, vol. 217, no. 17, pp. 7324-7348, 2011.

[18] R. Sharma, R. Bhargava, and I. V. Singh, “Combined effect of magnetic field and heat absorption on unsteady free convection and heat transfer flow in a micropolar fluid past a semi-infinite moving plate with viscous dissipation using element free Galerkin method," Applied Mathematics and Computation, vol. 217, no. 1, pp. 308-321, 2010.

[19] M. Sajid, R. Mahmood, and T. Hayat, "Finite element solution for flow of a third grade fluid past a horizontal porous plate with partial slip," Computers and Mathematics with Applications, vol. 56, no. 5 , pp. 1236-1244, 2008.

[20] Z. Wang and B. Hu, "Research of combined hybrid method applied in the Reissner-Mindlin plate model," Applied Mathematics and Computation, vol. 182, no. 1, pp. 49-66, 2006.

[21] C.-S. Chen, C.-P. Fung, and R.-D. Chien, "A further study on nonlinear vibration of initially stressed plates," Applied Mathematics and Computation, vol. 172, no. 1, pp. 349-367, 2006.

[22] A. Benjeddou and J. F. Deü, "A two-dimensional closed-from solution for the free-vibrations analysis of piezoelectric sandwich plates," International Journal of Solids and Structures, vol. 39, no. 6, pp. 1463 1486, 2002.

[23] V. M. Franco Correia, M. A. Aguiar Gomes, A. Suleman, C. M. Mota Soares, and C. A. Mota Soares, "Modelling and design of adaptive composite structures," Computer Methods in Applied Mechanics and Engineering, vol. 185, no. 2-4, pp. 325-346, 2000. 
[24] P. Heyliger and D. A. Saravanos, "Exact free-vibration analysis of laminated plates with embedded piezoelectric layers," Journal of the Acoustical Society of America, vol. 98, no. 3, pp. 1547-1557, 1995.

[25] H. J. Ding and W. Q. Chen, Three Dimensional Problems of Piezoelectricity, Nova Science Publishers, New York, NY, USA, 2001.

[26] D. A. Saravanos, P. R. Heyliger, and D. A. Hopkins, "Layerwise mechanics and finite element for the dynamic analysis of piezoelectric composite plates," International Journal of Solids and Structures, vol. 34, no. 3, pp. 359-378, 1997.

[27] K. Y. Lam, X. Q. Peng, G. R. Liu, and J. N. Reddy, “A finite-element model for piezoelectric composite laminates," Smart Materials and Structures, vol. 6, no. 5, pp. 583-591, 1997.

[28] X. Q. He, T. Y. Ng, S. Sivashanker, and K. M. Liew, "Active control of FGM plates with integrated piezoelectric sensors and actuators," International Journal of Solids and Structures, vol. 38, no. 9, pp. 1641-1655, 2001.

[29] M. A. Ramos Loja, C. M. Mota Soares, and C. A. Mota Soares, "Higher-order B-spline finite strip model for laminated adaptive structures," Composite Structures, vol. 52, no. 3-4, pp. 419-427, 2001.

[30] M. A. R. Loja, J. Infante Barbosa, C. M. Mota Soares, and C. A. Mota Soares, "Analysis of piezolaminated plates by the spline finite strip method," Computers and Structures, vol. 79, no. 26-28, pp. 23212333, 2001.

[31] O. C. Zienkiewicz and R. L. Taylor, The Finite Element Method, McGraw-Hill, New York, NY, USA, 4th edition, 1989.

[32] A. Houmat, "Triangular Fourier $p$ element for the analysis of membrane vibrations," Journal of Sound and Vibration, vol. 230, no. 1, pp. 31-43, 2000.

[33] K. S. Woo, C. H. Hong, P. K. Basu, and C. G. Seo, "Free vibration of skew Mindlin plates by p-version of F.E.M," Journal of Sound and Vibration, vol. 268, no. 4, pp. 637-656, 2003.

[34] A. Y. T. Leung and B. Zhu, "Comments on 'Free vibration of skew Mindlin plates by $\rho$-version of F.E.M.'," Journal of Sound and Vibration, vol. 278, no. 3, pp. 699-703, 2004.

[35] A. Y. T. Leung and J. K. W. Chan, "Fourier $p$ element for the analysis of beams and plates," Journal of Sound and Vibration, vol. 212, no. 1, pp. 179-185, 1998.

[36] A. Y. T. Leung, B. Zhu, J. Zheng, and H. Yang, "Analytic trapezoidal Fourier $p$ element for vibrating plane problems," Journal of Sound and Vibration, vol. 271, no. 1-2, pp. 67-81, 2004.

[37] A. Y. T. Leung, B. Zhu, J. Zheng, and H. Yang, "A trapezoidal Fourier $p$ element for membrane vibrations," Thin-Walled Structures, vol. 41, no. 5, pp. 479-491, 2003.

[38] A. Y. T. Leung and B. Zhu, "Transverse vibration of thick polygonal plates using analytically integrated trapezoidal Fourier $p$ element," Computers and Structures, vol. 82, no. 2-3, pp. 109-119, 2004.

[39] A. Y. T. Leung, C. Xiao, B. Zhu, and S. Yuan, "Free vibration of laminated composite plates subjected to in-plane stresses using trapezoidal $p$ element," Composite Structures, vol. 68, no. 2, pp. 167-175, 2005.

[40] A. Houmat, "Hierarchical finite element analysis of the vibration of membranes," Journal of Sound and Vibration, vol. 201, no. 4, pp. 465-472, 1997.

[41] J. N. Reddy, Mechanics of Laminated Composite Plates: Theory and Analysis, CRC Press, New York, NY, USA, 1996.

[42] H. J. Ding, W. Q. Chen, and R. Q. Xu, "New state space formulations for transversely isotropic piezoelasticity with application," Mechanics Research Communications, vol. 27, no. 3, pp. 319-326, 2000. 


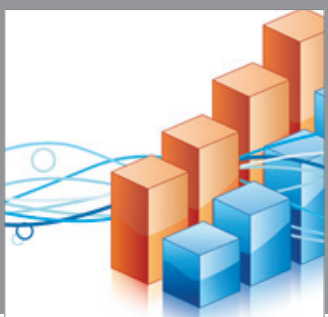

Advances in

Operations Research

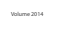

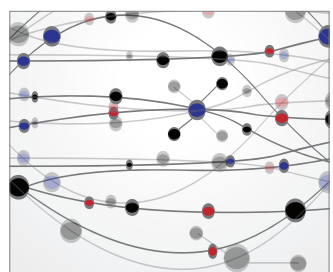

\section{The Scientific} World Journal
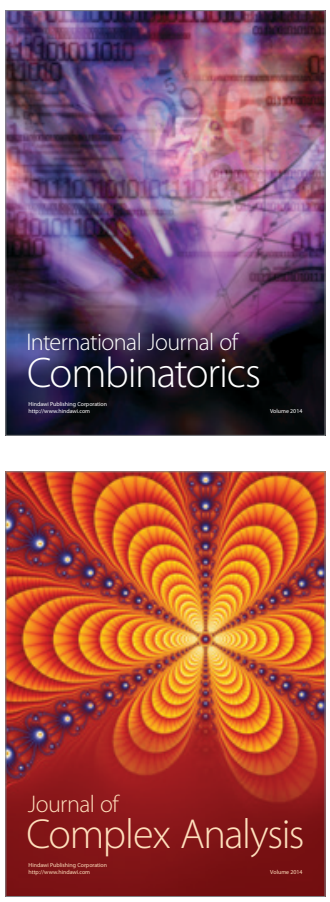

International Journal of

Mathematics and

Mathematical

Sciences
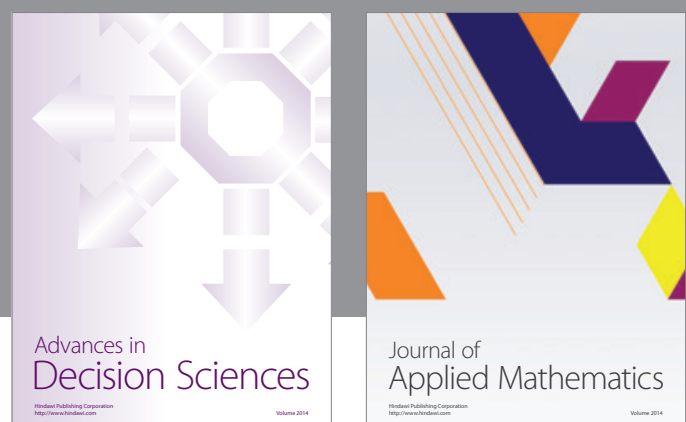

Journal of

Applied Mathematics
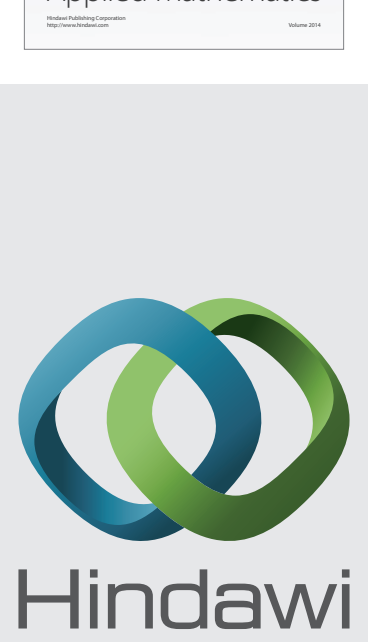

Submit your manuscripts at http://www.hindawi.com
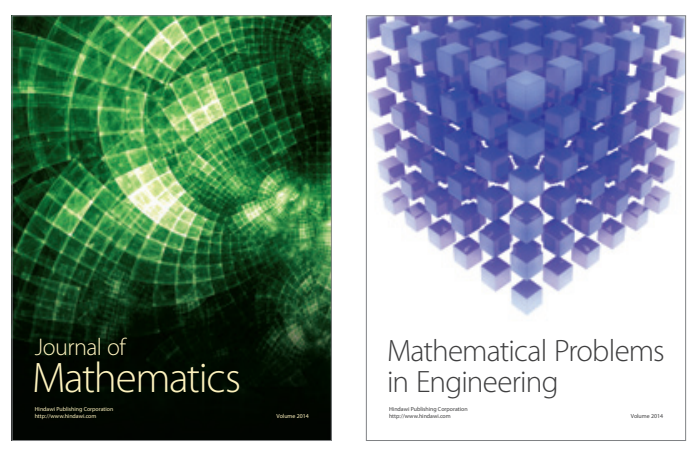

Mathematical Problems in Engineering
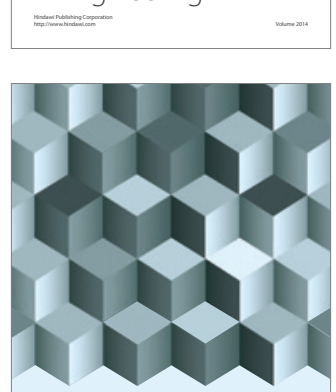

Journal of

Function Spaces
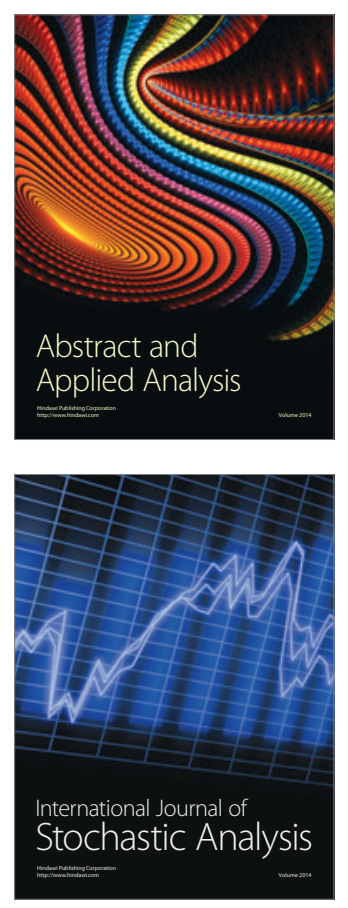

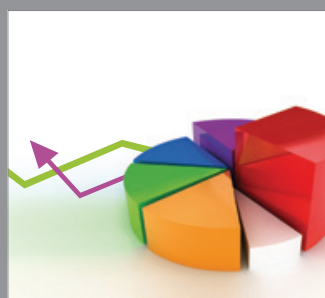

ournal of

Probability and Statistics

Promensencen
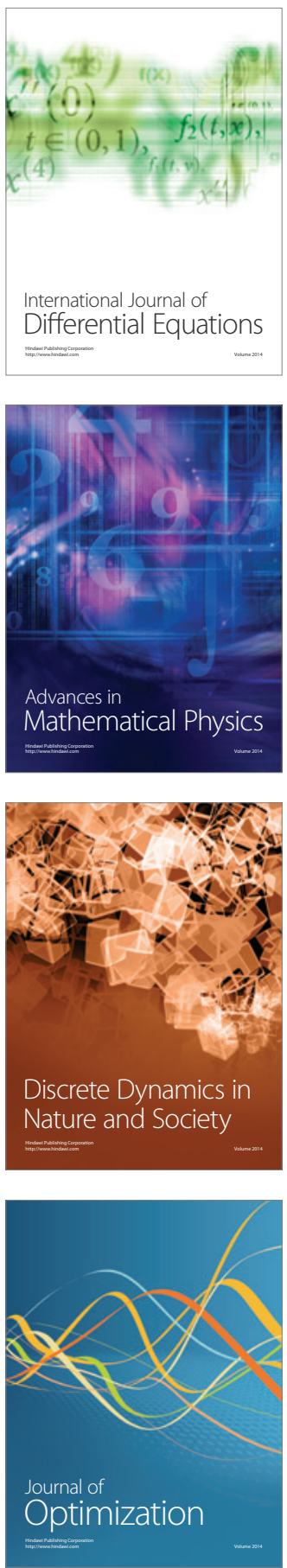